\title{
Psicologia da Saúde e Psicologia Positiva: Perspectivas e Desafios
}

Health psychology and positive psychology:

Perspectives and challenges

Prisla Ücker

Calvetti,Marisa Campio Muller \& Maria Lúcia Tiellet Nunes

Pontifícia Universidade

Católica do

Rio Grande do Sul

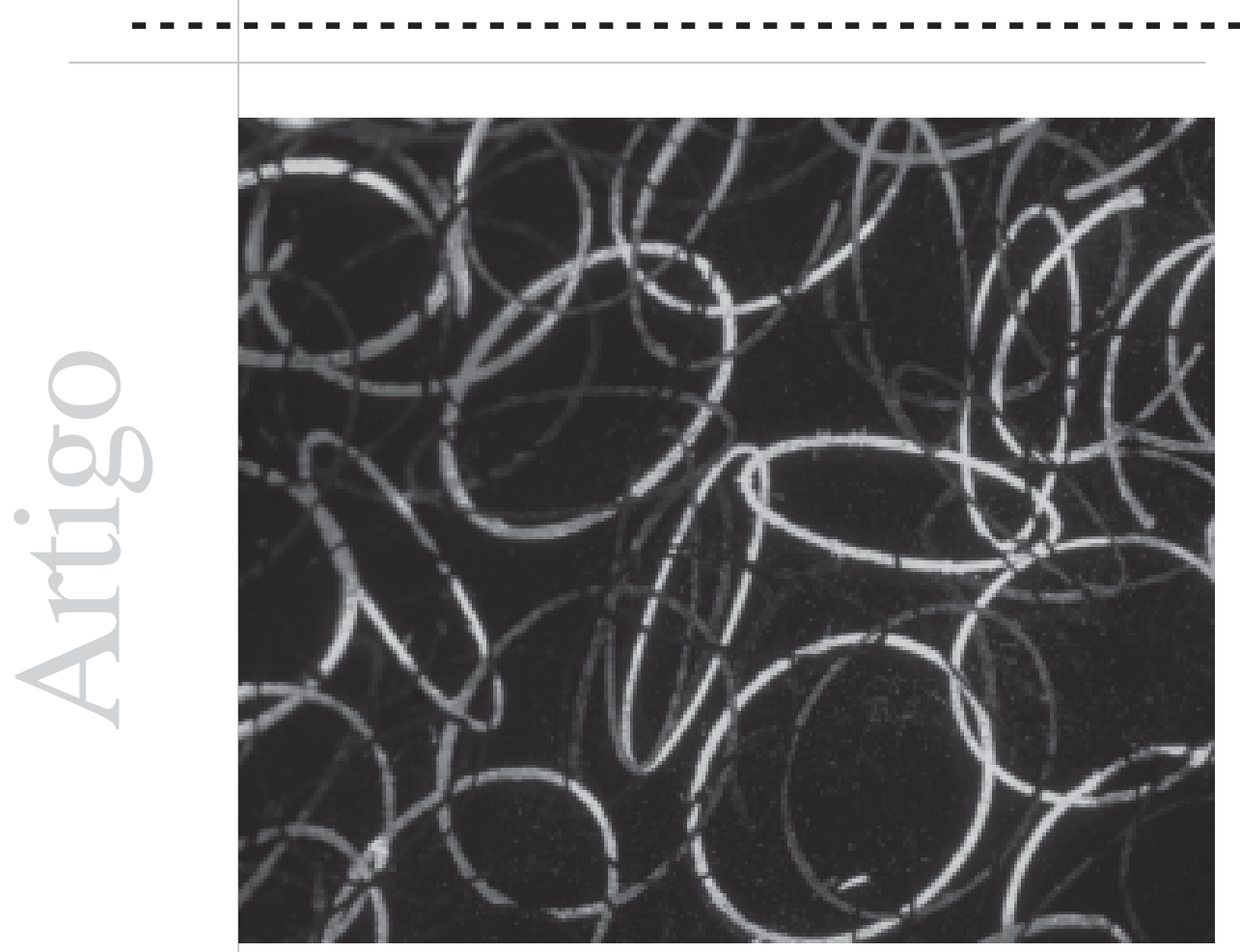




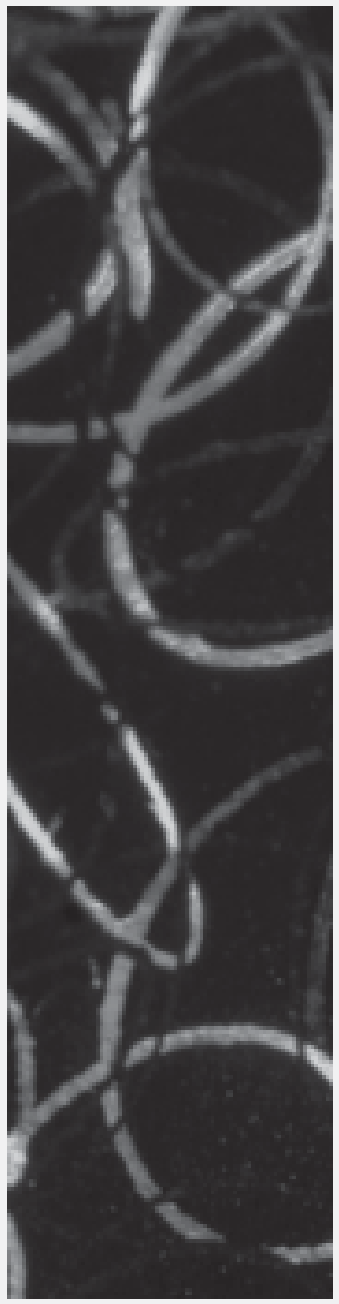

Resumo: O presente artigo visa a discutir a interface entre Psicologia da saúde e Psicologia positiva, destacando a relevância dos aspectos positivos do desenvolvimento humano como foco de pesquisas e intervenções. Salienta-se a necessidade de avanços nos estudos relacionados aos fatores protetores e de manutenção do desenvolvimento humano saudável. Na discussão em foco neste artigo, a Psicologia da saúde e a Psicologia positiva têm importante papel na compreensão dos aspectos envolvidos no enfrentamento da doença bem como na manutenção da saúde da pessoa, e apontam a relevância do investimento científico na investigação dos fatores de proteção da saúde.

Palavras-chave: Psicologia da saúde, Psicologia positiva, proteção da saúde.

Abstract: This study aims to discuss the aspects related to spirituality and quality of life in the health context. It encloses a discussion around the interface between Health Psychology and Positive Psychology, standing out the importance of positive variables as research and intervention focus in health. It is important to point out the necessity of advances in studies related to protection factors and the healthy human development maintenance. In the focused discussion, Health Psychology and Positive Psychology have an important role for the comprehension of the aspects involved in sickness confrontation as well as for people's health maintenance.

Key words: Health Psychology, Positive Psychology, health protection.
Este artigo tem por objetivo discutir a interface da Psicologia da saúde e Psicologia positiva no processo saúde-doença. A partir do entendimento dessa interface, reflete perspectivas e desafios para profissionais psicólogos e pesquisadores acerca de estudos e intervenções na atenção primária, secundária e terciária. Além disso, propõe a discussão acerca dos fatores de proteção da saúde, além da relevância dos estudos relacionados aos fatores de risco, no intuito de serem esses aspectos inseridos no planejamento e na implementação de ações em saúde pública.

\section{Contribuições da Psicologia da saúde}

Joseph Matarazzo (1980) refere-se à Psicologia da saúde como uma área de contribuições profissionais, científicas e educacionais da Psicologia para a promoção e a manutenção da saúde; assim, visa à prevenção e ao tratamento do processo saúde-doença e à identificação dos fatores relacionados ao desenvolvimento de enfermidades, além de buscar contribuir para a análise e a melhora do sistema dos serviços de saúde e para a elaboração de uma política sanitária. 
A Psicologia da saúde trata de um campo de natureza interdisciplinar que tem por finalidade realizar estudos relacionados à promoção, prevenção e tratamento da saúde do indivíduo e da população para a melhoria da qualidade de vida. (Remor, 1999; Brannon, Feist, 2001). Essa área centra-se na atenção primária, secundária e terciária e aspira dedicar-se, no futuro, à promoção e à educação para a saúde. (Castro, Bornholdt, 2004). As pesquisas e as intervenções em Psicologia da saúde têm integrado seus resultados nos cuidados de pacientes em uma variedade de estudos clínicos. (Nicassio, Meyerowitz, 2004).

A Divisão de Psicologia da Saúde da American Psychological Association (APA) estabeleceu objetivos para a Psicologia da saúde, dentre os quais se destacam: compreender e avaliar a interação existente entre o estado de bemestar físico e os diferentes fatores biológicos, psicológicos e sociais: buscar entender como as teorias e os métodos de pesquisa psicológica podem ser aplicados para potencializar a promoção da saúde e o tratamento da doença. (Remor, 1999).

Nesse sentido, evidencia-se a importância da integração do profissional psicólogo com outros profissionais da área da saúde, visto que são diversos os fatores relacionados no processo saúde-doença. Keefe e Blumenthal (2004) destacam que o futuro da Psicologia da saúde está em ampliar o desenvolvimento do modelo biopsicossocial, isso porque os fatores psicossociais têm se mostrado presentes em pesquisas relacionadas às intervenções em relação ao processo saúdedoença. Dessa forma, faz-se necessário propor atividades de prevenção e tratamento bem como a realização de programas de treinamento sobre as emergências desse campo na busca da melhoria da qualidade de vida.

O modelo biopsicossocial, de acordo com Straub (2005), explica que os comportamentos se caracterizam por processos biológicos, psicológicos e sociais. Esse modelo enfatiza a influência mútua entre esses aspectos no desenvolvimento humano, fundamentando-se na teoria sistêmica do comportamento, que compreende que o corpo é formado por sistemas em interação, como o endócrino, o cardiovascular, o nervoso e o imunológico, que interagem com os aspectos psicossociais.

Os psicólogos da saúde utilizam o modelo biopsicossocial em inúmeras áreas, incluindo HIV/AIDS, adesão a regimes de tratamento médico e efeitos de variáveis psicológicas e sociais sobre o funcionamento imunológico no processo saúde-doença. Essa perspectiva biopsicossocial foi utilizada primeiramente para explicar problemas de saúde.

Autores como Castro e Bornholdt (2004) destacam que, historicamente, a Psicologia da saúde começou a consolidar-se em 1970, nos EUA, através da American Psychological Association (APA), que criou a primeira associação de grupo de trabalho na área de saúde. Em 1979, cria a Divisão 38, a Health Psychology. A APA publica, desde 1982, a revista Health Psychology, a primeira na área. Na Europa, em 1986, teve início a European Health Psychology Society (EHPS). Desde então, foram criados outros periódicos na área, dentre eles, o British Journal of Health Psychology (Reino Unido); na Espanha, a revista Psicología de la Salud; na Itália, a Psicologia della Salute, e, na Bélgica, Gedrag \& Gezondheid. 
Em Portugal, a Sociedade Portuguesa de Psicologia da Saúde (SPPS) tem tido forte atuação e prestígio, e tem desenvolvido congressos nacionais, com publicação de revista na área, intitulada Psicologia Saúde \& Doenças. Atualmente, também existem programas de pós-graduação em Psicologia da saúde, além de linhas de pesquisas específicas nas diversas regiões do país. Em outros países da Europa, especificamente na Espanha e na Inglaterra, encontram-se programas de pós-graduação em Psicologia da saúde.

No Brasil, a Associação Nacional de Pesquisa e Pós-Graduação em Psicologia (ANPEPP) é constituída por grupos de trabalhos, dentre estes, o de Psicologia da saúde, em instituições e na comunidade. Pode-se destacar que, no País, existem diversas linhas de pesquisa relacionadas à área da saúde na Psicologia, porém existe apenas um programa de pós-graduação em Psicologia da saúde, o da Universidade Metodista de São Paulo. Os encontros da ANPEPP têm o objetivo de propiciar a reunião de pesquisadores para a elaboração de planos de ações conjuntas, definições de temas e publicações de livros e revistas, dentre outras formas de multiplicação e aprofundamento de pesquisas.

Foi realizado na cidade de Faro, em Portugal, no ano 2006, o $6^{\circ}$ Congresso Nacional de Psicologia da Saúde, que teve como tema saúde, bem-estar e qualidade de vida no foco das discussões dos profissionais e pesquisadores da área. Dentre os temas, destacou-se o da interface da Psicologia da saúde com a Psicologia positiva, chamandose a atenção dos pesquisadores para os aspectos positivos do desenvolvimento humano bem como para a necessidade de maior ênfase na promoção e prevenção em saúde, além de questões relacionadas ao tratamento de enfermidades para a melhoria da qualidade de vida. (Silva, 2006).

Pode-se observar a existência de uma nova direção para as pesquisas e intervenções em Psicologia da saúde, internacionalmente e no Brasil. O grupo de trabalho da ANPEPP tem tido como perspectivas e desafios da pósgraduação brasileira a realização de um planejamento formado pelo enfoque dos seguintes temas: intervenções eficientes baseadas em evidências e com boa relação custo-benefício, estudo de fatores de risco para problemas de saúde e participação na formação de médicos e de outros profissionais de saúde, visando à promoção da saúde na formação de futuros profissionais.

\section{Contribuições da Psicologia positiva}

Surge em 1998, com Martin Seligman, a discussão acerca dos fatores de proteção da saúde na Psicologia positiva. Dentre as variáveis positivas, tem-se estudado, recentemente, o otimismo, a espiritualidade, a criatividade e a imagem corporal, que têm sido associados ao bem-estar e à qualidade de vida de pessoas doentes e não-doentes e também a seus cuidadores. (SILVA, 2006).

Tem crescido o número de escolas da Psicologia no âmbito internacional que vêm se interessando tanto pelos aspectos de bemestar e saúde, quanto pelos aspectos negativos, como o estresse e a doença. Seligman e Czikszentmihalyi (2000), bem como Aspiwall e Staudinger (2003), ressaltam que a Psicologia científica, por muitos anos, foi focada desproporcionalmente na patologia e na reparação do dano. Segundo esses autores, é necessária também a atenção aos aspectos sadios do desenvolvimento humano.
Tem crescido o número de escolas da Psicologia no âmbito internacional que vêm se interessando tanto pelos aspectos de bem-estar e saúde, quanto pelos aspectos negativos, como o estresse e a doença. 
Em relação às contribuições da Psicologia positiva para a Psicologia clínica, Seligman e Peterson (2003) destacam os seguintes aspectos relacionados à resiliência e às forças no desenvolvimento humano: sabedoria e conhecimento, coragem, amor, justiça e transcendência. Salientam também a importância na ênfase da prevenção no processo de psicoterapia como, por exemplo, a atenção à conotação otimista como forma de prevenir a depressão e a ansiedade em crianças e adultos. A prática da Psicologia positiva transcende o sistema de saúde vigente; propõe estimular o desenvolvimento das forças positivas inerentes à pessoa e sugere o investimento em intervenções nesse enfoque.

A Psicologia positiva tem sido um movimento dos pesquisadores para o enfoque nos aspectos potencialmente sadios da pessoa. Conforme Yunes (2003), a resiliência tem tido destaque por estar relacionada a processos que buscam compreender a superação de adversidades. O autor também salienta a necessidade do avanço nas pesquisas relacionadas ao tema quanto aos aspectos de saúde.

A partir da compreensão da interação da pessoa com o ambiente, é possível ampliar o entendimento dos fatores de risco e de proteção envolvidos no processo saúdedoença. Os fatores de risco estão relacionados aos eventos negativos que aumentam a probabilidade de a pessoa apresentar problemas físicos, psicológicos e sociais. Os fatores de proteção se referem às influências que transformam ou melhoram respostas pessoais. Morais e Koller (2004) discutem a resiliência como variável de proteção à saúde. As autoras afirmam que houve um avanço em pesquisas sobre prevenção sob uma perspectiva da construção de aspectos positivos, que incluem resiliência, bem-estar subjetivo, otimismo, felicidade, autodeterminação, esperança, criatividade, habilidades interpessoais e fé.

A história da Psicologia positiva teve início com Martin Seligman, em 1998, enquanto estava no cargo de presidente da American Psychological Association (APA). Esse campo de pesquisa e intervenção destaca-se em relação a diversas variáveis subjetivas, como bem-estar e satisfação, esperança e otimismo e felicidade. (Linley et al., 2006). A Psicologia positiva tem se consolidado recentemente, e publicou seu primeiro periódico oficial na área no ano 2006, intitulado Journal of Positive Psychology.

Historicamente, conforme destaca Giacomoni (2002) em sua tese de doutorado, a Psicologia apresenta as suas primeiras pesquisas em relação à perspectiva positiva em alguns estudos, dentre eles, o de Carl Gustav Jung sobre a preocupação com o sentido da vida e os da Psicologia humanista na década de 1960, que tem como um dos seus principais pensadores Carl Rogers e Abraham Maslow. Torna-se importante destacar que Maslow, em 1954, escreveu um capítulo intitulado Toward a Positive Psychology, no livro Motivação e Personalidade.

Seligman e Csikszenmihalyi (2000) destacam que a Psicologia positiva traz para este novo século contribuições para o entendimento científico e para intervenções em indivíduos, famílias e comunidades. Entendem, ainda, que essa área não é um novo conceito, mas que sugere um redirecionamento do enfoque das pesquisas e intervenções para os aspectos sadios do desenvolvimento. 
No Brasil, em busca em bases de dados na LILACS, no período de 2000 a junho de 2006, encontram-se poucos estudos relacionados ao tema da resiliência, que se aproximam de uma perspectiva da Psicologia positiva, ainda não em destaque. Também são escassas as pesquisas referentes a variáveis positivas, como, por exemplo, religiosidade, espiritualidade, otimismo e esperança.

Não existe, até o ano 2006, um grupo de trabalho na ANPEPP em relação à Psicologia positiva, porém, nesse mesmo ano, foi publicado, em encontro realizado em Florianópolis, o livro Resiliência e Psicologia Positiva (Dell’Aglio, Koller, Yunes, 2006). Pode-se observar um movimento que tem iniciado a consolidação dessa área.

\section{Perspectivas e desafios na interface entre Psicologia da saúde e Psicologia positiva}

A Organização Mundial de Saúde (OMS) define saúde como o estado de completo bem-estar físico, mental e social, e, desde 1983, discute a inclusão da dimensão espiritual. Nesse âmbito, entende-se qualidade de vida como a harmonização de diferentes modos de viver e dos níveis físico, mental, social, cultural, ambiental e espiritual. (Fleck et al., 2003).

Existem dois aspectos conceituais que estão diretamente relacionados ao termo qualidade de vida: subjetividade e a multidimensionalidade, de acordo com Seidl e Zannon (2004). O primeiro refere-se à percepção da pessoa sobre o seu estado de saúde e sobre os aspectos não médicos do seu contexto de vida. O segundo aspecto se relaciona ao fato de que o referido construto é composto de variadas dimensões, e a identificação destas tem sido objeto de pesquisa científica em estudos empíricos.

O aspecto espiritualidade tende a ser visto como uma dimensão da natureza humana relacionada à qualidade de vida. (Marques, 2000). Esse construto multidimensional, avaliado em relação à percepção que o indivíduo tem sobre o seu bem-estar geral e sobre os seus estilos de vida, também pode levá-lo a valorar o sentido de sua vida. O estudo sobre qualidade de vida permite que se visualizem os aspectos da vida da pessoa que estão mais saudáveis e os que estão mais prejudicados. Dessa forma, resultados de investigação podem auxiliar um planejamento de intervenção psicossocial, no intuito de promover a saúde.

A literatura científica internacional apresenta trabalhos nos quais são explorados aspectos de espiritualidade no tratamento de doenças crônicas, melhora de quadros clínicos graves e no restabelecimento pós-cirúrgico. (Culloford, 2002). A literatura também apresenta um grande número de investigações que relacionam a espiritualidade à qualidade de vida (Hill, Pargament, 2003) bem como o desenvolvimento de inventários de espiritualidade. (Ellison, 1983; EllerhorstRyan, 1998; Fee, Ingran, 2004; Panzini, Bandeira, 2005).

Nesse âmbito, torna-se fundamental diferenciar e esclarecer o entendimento sobre religiosidade, espiritualidade e crenças pessoais. A religiosidade refere-se à extensão em que um indivíduo acredita, segue e pratica uma religião. Espiritualidade tem sido entendida como as questões relacionadas ao significado da vida e da razão de viver,
O aspecto espiritualidade tende a ser visto como uma dimensão da natureza humana relacionada à qualidade de vida. (Marques, 2000). 
independentemente de crenças e práticas religiosas. Já as crenças pessoais são crenças ou valores que a pessoa sustenta e que formam a base de seu estilo de vida e de comportamento. O construto espiritualidade torna-se central para a avaliação em saúde ao oferecer um referencial de significados para o enfrentamento da enfermidade. (Fleck et al., 2003).

No contexto das práticas de saúde, a influência de aspectos religiosos na cura e no tratamento de doenças tem sido pesquisada por Faria e Seidl (2005) como uma forma de enfrentamento destacada para lidar com situações de adversidade, como a situação de enfermidade. Os autores salientam a importância da escuta dos aspectos religiosos bem como dos demais aspectos do desenvolvimento humano no contexto do atendimento psicológico.

No Brasil, também já existem estudos sobre espiritualidade realizados e publicados, porém ainda são escassos, com destaque daqueles relativos à validação de instrumentos e ao processo saúde-doença. (Volcan et al., 2003; Fleck et al., 2003; Panzini, Bandeira, 2005; Faria, Seidl, 2005). Uma pesquisa bibliográfica identificou muitos instrumentos referentes ao tema da religiosidade e espiritualidade; estes, entretanto, em sua maioria, são do âmbito internacional. Nunes (2005) encontrou um total de dezessete instrumentos na busca realizada no período de 2000 a 2003 na base de dados PsycINFO. Enfatiza a autora a importância da validação de instrumentos para o contexto brasileiro. Dentre os instrumentos validados para o Brasil sobre o tema da religiosidadeespiritualidade, encontra-se a escala de bemestar espiritual (SWBS), de Paloutizian e
Ellison (1982), e a versão brasileira validada por Volcan et al. (2003). O bem-estar espiritual refere-se à percepção subjetiva da pessoa em relação à sua crença, e é constituído por dois componentes: o bemestar religioso, que é relativo a Deus, e o bem-estar existencial, relacionado ao sentido de propósito e satisfação de vida. (ElLison, 1983).

Outros instrumentos relacionados à espiritualidade foram validados recentemente para o Brasil, dentre eles, a escala de coping religioso espiritual. (Panzini, Bandeira, 2005). Além deste, também tem sido desenvolvido, pela Organização Mundial de Saúde (OMS), a versão para o português do WHOQOL-SRPB - módulo espiritualidade, religiosidade e crenças pessoais. (Fleck et al., 2003).

Estudo realizado no contexto de saúde em relação ao aspecto bem-estar espiritual e apoio social em mulheres com câncer de mama apontou escores mais altos de bemestar espiritual religioso em relação ao bemestar existencial. Nesse estudo, realizado por Hoffmann (2006), o aspecto religioso foi entendido também como fonte de apoio social no enfrentamento da adversidade, a doença. Em outro estudo sobre o tema, Marques (2000) investigou a saúde e o bemestar espiritual em adultos em uma amostra não-clínica de quinhentas e seis pessoas. A análise estatística revelou que existe uma correlação altamente significativa entre espiritualidade e saúde geral, o que demonstra que essa variável pode contribuir para a promoção de saúde e a prevenção de doença.

No contexto de saúde, a identificação das situações de estresse e enfrentamento ou 
coping vem sendo foco de pesquisas. Esse aspecto tem sido entendido como a adaptação psicossocial do ser humano diante da adversidade, influenciada pelas exigências situacionais, limitações, recursos disponíveis e fatores pessoais relacionados ao significado que a pessoa atribuir ao contexto. O coping religioso, em pessoas com HIV/AIDS, foi relacionado a escores mais baixos de depressão em estudo realizado por Woods et al. (1999).

Pesquisas revelam que a espiritualidade possui relação estreita com a melhora da qualidade de vida de pacientes com doenças crônicas. (Volcan et al., 2003). Estudo realizado com pacientes portadores de HIV/AIDS revelou que aqueles que apresentavam escores mais elevados de bem-estar espiritual tendiam a ser mais esperançosos. (Fleck et al., 2003).

A inter-relação entre o bem-estar espiritual e a saúde geral, em estudo realizado também por Marques (2000), mostra importantes associações entre espiritualidade e saúde geral. A autora atribui relevância à inclusão dessa dimensão na concepção de saúde, integrada às dimensões biológica, psicológica e social, pois ela contribui para a promoção da saúde e a prevenção de doenças bem como para o bem-estar das pessoas.

Siegel e Schrimshaw (2002), em uma pesquisa realizada em Nova York com sessenta e quatro portadores do HIV, relatam que as crenças espirituais e religiosas podem auxiliar na descoberta de significado na experiência da doença e no manejo do estresse. Os participantes dessa pesquisa mostraram uma variedade de benefícios advindos da religiosidade e espiritualidade, como o conforto das emoções e dos sentimentos, força e controle, suporte emocional e senso de pertença, encontro de significado e aceitação da doença, com redução da culpa.

A resiliência tem tido destaque como importante processo de proteção à saúde. (Rutter, 1990; Pesce et al., 2004; Yunes, 2003). Esse conceito se refere, conforme Pinheiro (2004), à capacidade que o indivíduo possui para se recuperar psicologicamente frente às adversidades, tendo como características a flexibilidade e a versatilidade, e advém do significado que a pessoa atribui aos eventos da vida, da capacidade afetiva, e, ainda, da presença de projeto de vida, e tem enfoque positivo no desenvolvimento humano.

De acordo com Yunes e Szymanski (2001), a resiliência possui características individuais e ambientais. No entendimento desse mecanismo, o indivíduo e o ambiente são vistos como sistemas de formação mútuos. As autoras frisam a importância dessa contribuição para o planejamento e as intervenções em políticas públicas.

Walsh (1998) afirma que a resiliência em famílias é constituída pelos seguintes processos: sistemas de crenças, padrões de organização e de comunicação. O primeiro refere-se à atribuição de sentido à adversidade, ao olhar positivo, à transcendência e à espiritualidade; o segundo representa a flexibilidade, a coesão e os recursos sociais e econômicos, e o terceiro processo relaciona-se à clareza, às expressões emocionais abertas e à colaboração na solução de problemas. A partir disso, podese observar que a espiritualidade se relaciona ao processo de resiliência, sendo um dos aspectos que contribui para o aprendizado e o crescimento através da adversidade. 
A associação entre positividade e crenças religiosas e espirituais foi demonstrada em estudo realizado por Sodergren et al. (2004) com cento e noventa e quatro pacientes com problemas respiratórios. Os autores salientam a necessidade de estudos que aprofundem a investigação sobre esta relação: a positividade tem mais fortemente relação com a religiosidade ou com as crenças espirituais? Hasnain et al. (2005) apontaram o fato de a religiosidade poder ser preventiva de comportamentos de risco, como o uso de drogas e de práticas sexuais sem proteção.

No que tange à espiritualidade e à psicoterapia, Nunes e Müller (2003) relatam que existe uma associação entre a dimensão da fé e a psicoterapia, afirmando que uma pode potencializar a outra e auxiliar, assim, na melhoria da qualidade de vida. Ressaltam que a espiritualidade pode ajudar a pessoa a enfrentar dificuldades referentes a determinada experiência de vida, porém destacam que ainda são frágeis as evidências sobre essa relação, sendo necessário o aumento das pesquisas na área.

Frente ao advento da Psicologia positiva, torna-se necessária a ampliação do modelo biopsicossocial da área da Psicologia da saúde, que inclui aspectos como, por exemplo, bemestar, felicidade, resiliência, coping, espiritualidade e apoio social no desenvolvimento de pesquisas e intervenções em saúde. A atenção dos pesquisadores para os aspectos positivos do desenvolvimento humano necessita ser incorporada em estudos e intervenções a fim de contribuir para o entendimento dos mecanismos de proteção da saúde.
Desde o advento da Psicologia da saúde, tem sido destacada a necessidade da ênfase não só na cura e no tratamento de doenças mas também na promoção e na prevenção da saúde. Porém, pode-se perceber que, mais recentemente, o movimento da Psicologia positiva tem fortalecido de fato esse aspecto, frisando a importância do foco no desenvolvimento sadio.

Em estudo realizado por Meneses (2006), através de revisão de literatura de artigos indexados na Psycarticles entre 2000-2005 com o descritor spirituality, a autora teve o objetivo de clarificar a importância do tema para a Psicologia da saúde e para a Psicologia positiva. Nesse estudo, a análise ratificou a inclusão dessa variável nas pesquisas em saúde bem como destacou o fato de ainda não se obter consenso em relação ao construto nas pesquisas científicas.

A investigação sobre o aspecto espiritualidade tem também contribuído para o entendimento dos fatores implicados na qualidade de vida. De acordo com Ribeiro (2006), um dos objetivos primários da Psicologia da saúde tem sido o resultado de melhoria da qualidade de vida, pois a intervenção em saúde visa a atuar para promover o bem-estar das pessoas.

A Psicologia da saúde que dá ênfase ao processo saúde-doença tem se referido à religiosidade e à espiritualidade como aspectos auxiliares no enfrentamento da doença. Nesse contexto, a Psicologia positiva tende a considerar essa dimensão como um dos aspectos formadores dos processos de resiliência, com destaque para a transcendência. Pode-se dizer que a religiosidade-espiritualidade contribui para a 
melhoria da qualidade de vida, por enfatizar os aspectos sadios do desenvolvimento humano.

Para o avanço do conhecimento e das práticas em Psicologia da saúde (Matarazzo, 1980) e Psicologia positiva (Seligman, 2000), tornase fundamental o entendimento dos seus aspectos individuais e ambientais na perspectiva de interação mútua. A resiliência tem sido estudada nessa perspectiva e podese pensar que a religiosidade e a espiritualidade tenham contribuído para esse processo, respectivamente, como fonte de apoio social e no sentido de ressignificar a doença frente à situação de adversidade. Esse mecanismo também pode contribuir para o enfrentamento do processo saúde-doença enquanto preditor de resultados positivos de saúde, como a qualidade de vida.

\section{Considerações finais}

Frente ao exposto, os aspectos psicológicos positivos têm sido recentemente estudados frente ao processo saúde-doença pelas áreas da Psicologia da saúde e também da Psicologia positiva. Pode-se entender a religiosidade-espiritualidade e a resiliência como aspectos protetores de saúde e de promoção da qualidade de vida. Pode-se observar que existe uma interface entre estas no contexto internacional. No Brasil, estudos realizados principalmente em relação à Psicologia positiva ainda não têm sido destacados, porém, nesse âmbito, evidenciam-se investigações sobre o processo de resiliência no desenvolvimento humano. A partir deste estudo, pode-se sugerir que as novas pesquisas relacionadas ao processo saúde-doença visem também a enfocar os aspectos sadios do desenvolvimento, como, por exemplo, as emoções positivas. Tornase necessário o investimento em intervenções na área da Psicologia a fim de contribuir para o planejamento das ações de saúde. Também se pode destacar a necessidade da atenção dos profissionais de saúde e pesquisadores, especialmente dos psicólogos, tanto para os fatores de proteção à saúde quanto para os fatores de risco, para os processos de resiliência em indivíduos e para os aspectos relacionados à vulnerabilidade.

Para tanto, faz-se necessário, para a formação do psicólogo nos currículos acadêmicos e de pós-graduação, no âmbito do ensino e no da pesquisa bem como no de intervenções em saúde, a inserção da discussão sobre os campos da Psicologia da saúde e da Psicologia positiva. Essas áreas possuem o intuito de contribuir para o avanço científico, chamando a atenção dos psicólogos para os aspectos sadios do desenvolvimento humano, como as emoções positivas. Destaca-se também a relevância de estudos relacionados aos mecanismos de risco e às emoções negativas, fundamentais para o aprofundamento do conhecimento científico no âmbito da Psicologia. Pretende-se apenas investir no avanço da perspectiva de promoção e de prevenção do processo saúde-doença em pesquisas e intervenções do psicólogo. Dessa forma, como compromisso do profissional na área, acredita-se contribuir também para o planejamento de ações de saúde pública. 


\section{Prisla Ücker Calvetti}

Psicóloga, Mestre em Psicologia Clínica pela Pontifícia Universidade Católica do Rio Grande do Sul (PUCRS), Especialista em Saúde Pública (ESP/RS-FIOCRUZ).

\section{Marisa Campio Muller}

Psicóloga, Doutora em Psicologia pela Pontifícia Universidade Católica de São Paulo (PUCSP), professora titular da Faculdade de Psicologia da PUCRS

\section{Maria Lúcia Tiellet Nunes}

Psicóloga, Doutora em Psicologia pela Universidade de Berlim-Alemanha, professora coordenadora do Programa de Pós-Graduação em Psicologia da Pontifícia Universidade Católica do Rio Grande do Sul (PUCRS).

Av. Princesa Isabel, 500, ap. 426 Bl. C2. Bairro Santana CEP 90620 000. Porto Alegre, RS. Fones: (51) 84394859 e (51) 32176890. E-mail: prisla.calvetti@gmail.com

ASPINWALL, L.; STAUDINGER, U. Apsychology of human strengths: some central issues of an emerging field. In: A psychology of human strengths: fundamental questions and future directions for a Positive Psychology. Washington: American Psychological Association, 2003. p. 9-22.

BRANNON, L.; FEIST, J. Psicología de la Salud. Madrid: Thomson Learning, 2001.

CASTRO, E., BORNHORD, E. Psicologia da saúde x Psicologia hospitalar: definições e possibilidades de inserção profissional. Psicologia: Ciência e Profissão, Brasília, v. 24, n. 3, p. 48-57, 2004.

CULLOFORD, L. Spirituality and clinical care. British Medical Journal, London, v. 325, p. 1434-1436, 2002.

DELL'AGLIO, D.D.; KOLLER, S. H.; YUNES, M.A.(Org.). Resiliência e Psicologia positiva: interfaces do risco à proteção. São Paulo: Casa do Psicólogo, 2006

ELLERHORST-RYAN, J. Instruments to measure spiritual status. In: Quality of life pharmacoeconomics in clinical trials, Filadélfia: Lippincott-Raven, Cap. 17, 1998. p. 145-153.
ELLISON, G. Spiritual well-being: conceptualization and measurement. Journal of Psychology and Theology, New York, v.11, n. 4, p. 330-340, 1983.

FARIA, J. B.; SEIDL, E. M. Religiosidade e enfrentamento em contextos de saude e doença: revisão de literatura. Psicologia, Reflexão e Crítica, Porto Alegre, v.18, n. 4, p. 381-389, 2005.

FEE, J.L.; INGRAM, J.A. Correlation of the Holy Spirit questionnaire with the spiritual well-being scale and the spiritual assessment inventory. Journal of Psychology and Theology, New York, v. 32, n. 2, p.104-115, 2004

FLECK, M.; BORGES, Z.; BOLOGNESI, G.; ROCHA, N. S. Desenvolvimento do WHOQOL, módulo espiritualidade, religiosidade e crenças pessoais. Revista de Saúde Pública, São Paulo, v. 37, n. 4 , p. 446-455, 2003.

GIACOMONI, C. Bem-estar subjetivo infantil: conceito de felicidade e construção de instrumentos para avaliação. Tese (doutorado em Psicologia do desenvolvimento). Programa de Pós-Graduação em Psicologia do Desenvolvimento da Universidade Federal do Rio Grande do Sul, Porto Alegre, 2002. 
HASNAIN, M.; SINACORE, M.; MENSAH,E.K.;LEVY, J.A. Influence of religiosity on HIV risk behaviors in active injection drug users. AIDS Care, Abingdon, v. 17, n.7, p. 892-901, 2005.

HILL, P.; PARGAMENT, K. Advances in the conceptualization and measurement of religion and spirituality. Implications for physical and mental health research. American Psychologist, New York, v.58, n. 1, p. 64-74, 2003.

HOFFMANN, F. Apoio social e bem-estar espiritual em mulheres: da espera pelo diagnóstico ao início do tratamento para cancer de mama. Dissertação (mestrado em Psicologia clínica). Programa de PósGraduação em Psicologia da Pontificia Universidade Católica do Rio Grande do Sul, 2006.

LINLEY, P.A; JOSEPH, S.; HARRINGTON, S.; WOOD, A. Positive Psychology: past, present and future. The Journal of Positive Psychology, Pensilvânia, v.1, n.1, p.3-16, 2006.

KEEFE, F;; BLUMENTHAL, J. Health Psychology: what will the future bring? Health Psychology, Washington, v. 23, n.2, p.156-157, 2004

MARQUES, L. F. A saúde e o bem-estar espiritual em adultos portoalegrenses. Tese (doutorado em Psicologia). Programa de PósGraduação em Psicologia da Pontifícia Universidade Católica do Rio Grande do Sul, Porto Alegre, 2000.

MASLOW, A.H. Motivation and personality. New York: Harper, 1954

MATARAZZO, J. Behavioural health's challenge to academic, scientific and professional Psychology. American Psychologist, New York, v. 37, 1980.

MENESES, R. O papel da espiritualidade na saúde, bem-estar e qualidade de vida. Trabalho apresentado no $6^{\circ}$ Congresso Nacional de Psicologia da Saúde, Faro, Portugal, 2006.

MORAIS, N.; KOLLER, S. Abordagem ecológica do desenvolvimento humano, Psicologia positiva e resiliência: ênfase em saúde. In: Ecologia do desenvolvimento humano: pesquisa e intervenção no Brasil. Silvia Helena Koller (Org.) São Paulo: Casa do Psicólogo, 2004. p. 91-107.

NICASSIO, P.; MEYEROWITZ, B. The future of Health Psychology interventions. Health Psychology, Washington, v. 23,n. 2,p. 132-137, 2004

NUNES, M. L.; MÜLLER, M. Espiritualidade influencia na qualidade de vida. PUCRS Informação, Porto Alegre, v. 26, n.116, p. 25, 2003.

NUNES, M. L. Instrumentos de pesquisa, espiritualidade, religiosidade. In: Ciência e Deus no mundo atual: uma abordagem inter e transdisciplinar. Marcos Volcan e Jovino Pizzi (Org.). Pelotas: EDUCAT, 2005. p. 278-284.

PALOUTIZIAN, R.; ELLISON, C. Loneliness, spiritual well-being and the quality of life. In: Loneliness: a sourcebook of current theory, research and therapy. PEPLAU, D.; PERLMAN, D. (Org.). New York: John Wiley and Sons, 1982. p. 224-235.

PANZINI, R. G;; BANDEIRA, D. Escala de coping religioso-espiritual (escala CRE): elaboração e validação de constructo. Psicologia em Estudo, Maringá, v.10, n.3, p.507-516, 2005.

PESCE, R.; ASSIS, S.; SANTOS, N.; OLIVEIRA, R. Risco e proteção: em busca de um equilíbrio promotor de resiliência. Revista Teoria e Pesquisa, Brasília, v. 20, n. 2, p.135-143, 2004.
PINHEIRO, D. A resiliência em discussão. Psicologia em Estudo, Maringá, v.9, n.1, p.67-75, 2004.

REMOR, E. Psicologia da saúde: apresentação, origens e perspectivas. Revista Psico, Porto Alegre, v. 30, n.1, p. 205-217, 1999.

RIBEIRO, J. Variáveis positivas e qualidade de vida ou percepção de saúde. Trabalho apresentado no $6^{\circ}$ Congresso Nacional de Psicologia da Saúde, Faro, Portugal, 2006.

RUTTER, M. Psychosocial resilience and protective mechanisms. In: Risk and protective factors in the development of psychopathology. ROLF, J.; MASTEN, A. S.; CICCHETTI, D.; NUECHTERLEIN, K. H. e WEINTRAUB, S. (Org.). Cambridge: Cambridge University Press, 1990. p. 181-214.

SELIGMAN, M.; CSIKSZENTMIHALYI, M. Positive Psychology: an introduction. American Psychologist, New York, v.55, n. 1, p. 5-14, 2000 .

SELIGMAN, M. Positive Psychology. In: The science of optimism \& hope: research essays in honor of Martin E. P. Seligman. GILHAM, J. E. (Org.). Pennsylvania: Templeton Foundation Press. Cap. 25, 2000. p. 415-429.

SELIGMAN, M.; PETERSON, C. Positive clinical Psychology. In: A Psychology of human strengths: fundamental questions and future directions for a positive Psychology. Cap. 21. ASPINWALL, L. G. e STAUDINGER, U. M. (Org.) Washington: American Psychological Association, 2003. p. 305-317.

SIEGEL, K.; SCHRIMSHAW, E. The perceived benefits of religious and spiritual coping among older adults living with HIV/AIDS. Journal for the scientific study of religion, Malden, v. 41, n. 1, p. 91-102, 2002.

SEIDL, E.; ZANNON, C. Qualidade de vida e saúde: aspectos conceituais e metodológicos. Cadernos de Saúde Pública, São Paulo, v. 20, n. 2, 2004, p. 580-582.

SILVA, I. Psicologia da saúde: uma perspectiva positiva. Trabalho apresentado no $6^{\circ}$ Congresso Nacional de Psicologia da Saúde, Faro, Portugal, 2006

SODERGREN, S.C.; HYLAND, M.E.; CRAWFORD, A.; PARTRIDGE M. R. Positivity in illness: self-delusion or existential growth. British Journal of Health Psychology, Londres, v. 9, p. 163-174, 2004.

STRAUB, R. Introdução à Psicologia da saúde. Cap. 1. In: Psicologia da saúde. Porto Alegre: Artmed, 2005. p. 21-51.

VOLCAN, S.; SOUSA, P.L.; MARI, J. J.; HORTA, B. L. Relação entre bem-estar espiritual e transtornos psiquiátricos menores: estudo transversal. Revista de Saúde Pública, São Paulo, v.37, n. 4, p. 440445, 2003.

YUNES, M. A.; SZYMANSKI, H. Resiliência: noção, conceitos afins e considerações críticas. In: Resiliência e educação. TAVARES, J. (Org.). São Paulo: Cortez, 2001. p.13-42.

YUNES, M. A. Psicologia positiva e resiliência: o foco no indivíduo e na família. Psicologia em Estudo, Maringá, v. 8, n.especial, 2003, p. 75-84.

WALSH, F. Strengthening family resilience. New York: London: The Guilford Press, 1998.

WOODS, T.E.; ANTONI, M. H.; IRONSON, G. H.; KLING, D.W. Religiosity is associated with affective and immune status in symptomatic HIV-infected gay men. Journal of Psychosomatic Research, v. 46, n. 2, 1999, p. 165-176. 